\title{
Energy dispersive spectroscopic measurement of charge traps in $\mathrm{MoTe}_{2}$
}

\author{
Nicola J. Townsend, ${ }^{1,2}$ Iddo Amit $\odot,{ }^{1,2, *}$ Vishal Panchal, ${ }^{3,4}$ Olga Kazakova, ${ }^{3}$ Monica F. Craciun, ${ }^{1}$ and Saverio Russo ${ }^{1, \dagger}$ \\ ${ }^{1}$ University of Exeter, College of Engineering, Mathematics, and Physical Sciences, Stocker Road, Exeter EX4 4QL, United Kingdom \\ ${ }^{2}$ Durham University, Department of Engineering, South Road, Lower Mountjoy, Durham DH1 3LE, United Kingdom \\ ${ }^{3}$ National Physical Laboratory, Teddington TW11 OLW, United Kingdom \\ ${ }^{4}$ Bruker Nano Surfaces UK, Coventry CV4 9GH, United Kingdom
}

(Received 2 May 2019; revised manuscript received 9 July 2019; published 31 October 2019)

\begin{abstract}
Spectroscopic techniques are vital to determine the energy distribution of trapped states in semiconducting materials to assess the quality and efficiency of electronic devices. However, there is a need for a sensitive spectroscopic technique that can be used with atomically thin materials which are thinner than the Debye screening length as the current methods, such as deep level trap spectroscopy and admittance spectroscopy, are incompatible with the long emission times and temperature sensitivities of these materials. In this work we expand the threshold voltage transient phenomenon into an energy dispersive spectroscopic technique, dubbed the threshold voltage transient spectroscopy technique. This is applied to few-layer $\mathrm{MoTe}_{2}$ with the trap concentration and subsequently the density of trap states found in a region between the valence band edge and the midgap, which clearly shows the density of states in the tail end of the valence band.
\end{abstract}

DOI: 10.1103/PhysRevB.100.165310

\section{INTRODUCTION}

Charge traps can have a significant impact on semiconductor device performance, manifesting in a large hysteresis and increased contact resistance through the formation of Schottky barriers. In devices based on two-dimensional (2D) semiconductors, this hysteresis is characterized by extraordinarily long transient times [1]. The phenomenon, which is most likely due to inefficient charge screening found in materials that are thinner than their Debye screening lengths [2,3], may limit the use of unencapsulated 2D materials in high-speed electronics, but can also prove to be advantageous in designing volatile memory applications [4,5] and light amplifiers [6-8]. The traps can hinder the efficiency of these devices, as they are thermally activated [9], so they exhibit a time-dependent response.

Spectroscopic measurements of the density of states (DOS) arising as a result of charge traps provide crucial information on semiconductor devices [10], which is used to determine whether to passivate their surface or eliminate the traps $[11,12]$. By identifying the energy distribution of the traps, it is possible to either exploit them or prevent them altogether. Therefore, a sensitive spectroscopic method suitable for determining the energy distribution of trap states in atomically thin materials is vital for assessing the quality and efficiency of freestanding, encapsulated [13], or defect-engineered [14]

\footnotetext{
*iddo.amit@durham.ac.uk

†s.russo@exeter.ac.uk
}

Published by the American Physical Society under the terms of the Creative Commons Attribution 4.0 International license. Further distribution of this work must maintain attribution to the author(s) and the published article's title, journal citation, and DOI. electronic components enabling their properties to be tailored for the desired application.

The two most common spectroscopic techniques for probing charge traps in conventional semiconductors are deep level trap spectroscopy (DLTS) [15] and admittance spectroscopy (AS) $[16,17]$. Both methods rely on the detection of thermally emitted carriers that are trapped within particular deep-level centers. The analysis is focused on nonradiative recombination processes, and is therefore based on Shockley-Read-Hall statistics $[18,19]$, which describes the capture and emission rates for the thermally activated processes. Optimized for bulk semiconductors, DLTS and AS operate at frequency ranges down to $\sim 1 \mathrm{kHz}$, and at temperatures lower than ambient [20-24]. However, we have recently shown that the process of charge capture and emission in 2D materials occurs at frequencies of $\sim 1 \mathrm{mHz}$ at room temperatures, rendering the traditional methods moot [1].

For semi-insulating materials and devices where the capacitance is too small to measure, current transient spectroscopy (CTS) provides efficient means to overcome the limitations of DLTS and AS [25]. The most notable method, current DLTS (I-DLTS), measures the variation in relaxation drain currents following a gate pulse, as a function of temperature [26]. As such, it cannot distinguish between trapped charges that are distributed across the channel and those that are concentrated at the metal-semiconductor interfaces.

Our previous study revealed that current transients contain crucial information that describes the dynamics of charge carriers in two-dimensional semiconductors, but are usually disregarded in I-DLTS. The approach, termed threshold voltage transients (TVTs), uses constant-temperature DC measurements to quantify time-resolved changes in the device threshold voltage [1]. Moreover, it holds the potential to extract the energy dispersion of trap states within the band gap. 
In this paper, we expand the threshold voltage transients into an energy dispersive spectroscopic technique. This method, which is a variant of current transient spectroscopy, is termed threshold voltage transient spectroscopy (TVTS), and is demonstrated on few-layer $\mathrm{MoTe}_{2}$ devices, in an energetic region between the valence band edge and the midgap. The resulting density of states clearly shows the trap concentration follows an expected decay trend as the energy shifts farther from the edge of the valence band. While there are no pronounced features arising from contaminants, only a small region of the midgap was probed, so they may be located out of the targeted range.

\section{EXPERIMENTAL TECHNIQUES}

$\mathrm{MoTe}_{2}$ was chosen given its wide applicability for infrared optoelectronic devices. Few-layer flakes were mechanically exfoliated onto a highly doped silicon substrate with $290 \mathrm{~nm}$ thick thermally grown oxide acting as a global back gate electrode and dielectric, respectively. The source and drain contacts were patterned using standard electron-beam lithography and metalized with $\mathrm{Ti} / \mathrm{Au}(5 / 50 \mathrm{~nm})$. The completed devices were then thermally annealed at $200{ }^{\circ} \mathrm{C}$ for two hours in a $\mathrm{H}_{2} / \mathrm{Ar}$ (10/90 percent) environment to remove any residual organic contamination.

Low-noise measurements were carried out in dark, ambient conditions. The drain electrode was biased using a low-noise source-measurement unit and the source electrode was kept grounded throughout the experiments, with the current flow being measured through the source electrode using a current preamplifier. An independent voltage sourcemeter was used to bias the gate electrode while simultaneously measuring the leakage current.

Kelvin probe force microscopy (KPFM) was performed with a Bruker Dimension DI 3100 in ambient conditions with both source and drain electrodes grounded, while biasing the gate. The measurements were done using a dual-pass ("lift mode") scan, in which the first scan records the topography of the sample in tapping mode, and the second scan records the contact potential difference $\left(V_{C}\right)$ at a constant vertical separation from the sample. The semiconductor work function $\left(\phi_{S}\right)$ is then extracted from the $V_{C}$ using the relation $V_{C}=\left(\phi_{t}-\phi_{S}\right) / q$, where $\phi_{t}$ is the work function of the AFM tip and $q$ is the elementary charge. $\phi_{S}$ was scanned on a constant line trace, while the gate electrode was slowly ramped, allowing a minimum of five scans per gate value.

\section{RESULTS AND DISCUSSION}

\section{A. Device electrical characteristics}

Indicative properties of the devices were extracted from their source-drain current vs source-drain bias $\left(I_{d s}-V_{d s}\right)$ response (see [27] for the $I_{d s}-V_{d s}$ response curve) and sourcedrain current vs gate bias $\left(I_{d s}-V_{g s}\right)$ transfer curves. The $I_{d s}-V_{g s}$ transfer curve taken at a constant $V_{d s}=3 \mathrm{~V}$, in Fig. 1, shows the expected behavior of a $p$-channel, accumulation-mode transistor. The charge carrier mobility was estimated using $\mu=L\left(d I_{d s} / d V_{g s}\right) /\left(W C_{o x} V_{d s}\right)$, with a channel length $L=$ $2.5 \mu \mathrm{m}$, width $W=4 \mu \mathrm{m}$, and gate oxide capacitance $C_{o x}=$ $\varepsilon_{r} \varepsilon_{0} / d_{o x}=115 \mu \mathrm{F} \mathrm{m}{ }^{-2}$, where $\varepsilon_{r}$ is the oxide permittivity

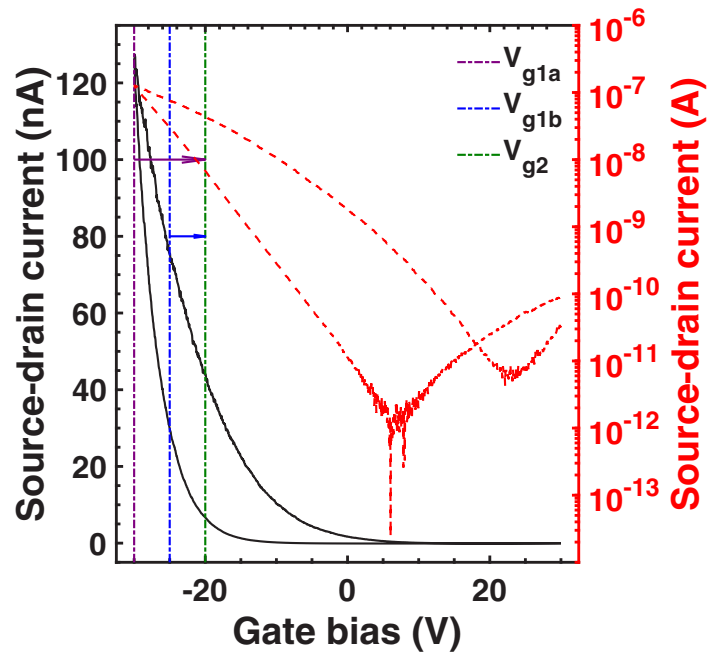

FIG. 1. The $I_{d s}-V_{g s}$ transfer curve of the same device taken with $V_{d s}=3 \mathrm{~V}$ shown on a linear (black) and semilogarithmic (red dashed) scale. The vertical lines indicate the pulse window used between $V_{g 1}$ and $V_{g 2}$ for two values of $V_{g 1}$ where $V_{g 1 a}<V_{g 1 b}$ with the arrows showing the emission from $V_{g 1}$ to $V_{g 2}$.

of 3.9 for $\mathrm{SiO}_{2}, \varepsilon_{0}$ is the vacuum permittivity, and $d_{o x}$ is the dielectric thickness of $290 \mathrm{~nm}$, and found to be approximately $0.44 \mathrm{~cm}^{2} \mathrm{~V}^{-1} \mathrm{~s}^{-1}$, which is comparable to that reported in earlier studies on two-terminal transistors [1]. The subthreshold swing, estimated using $S_{t h}=\left(d \log _{10} I_{d s} / d V_{g s}\right)^{-1}$, is found to be approximately $7 \mathrm{~V}$ decade $^{-1}$ for both the up- and the down-sweep. As discussed in [1], in these transistors $\mu$ and $S_{t h}$ are largely governed by the thermionic emission over the metal-semiconductor barriers, whereas the threshold voltage is directly dictated by the potential drop profile that forms between the gate and the channel. Since $\mu$ and $S_{t h}$ show little to no change between the two transfer sweep branches, in contrast to the threshold voltage $\left(V_{t h}\right)$, which changes by approximately $7 \mathrm{~V}$, it can be concluded that it is the ambientchannel-oxide dynamic interface that experiences the most significant changes.

The time constants of the traps can be extracted from time-resolved electrical measurements and a clear distinction can be made between TVT and contact emission transients. This involves pulsing the gate bias between the device "on" $\left(V_{g 1}\right)$ and "off" states $\left(V_{g 2}\right)$, and maintaining the gate bias to allow the current to saturate. The gate pulse measurements were carried out with $V_{d s}$ ranging between 3 and $5 \mathrm{~V}$, and the emission curves for each $V_{d s}$ truncated from the full cycle and fitted with a double exponential growth function of the form

$$
I_{d s}=I_{S}+A_{1} e^{-t / \tau_{1}}+A_{2} e^{-t / \tau_{2}},
$$

where $I_{S}$ is the saturation current once the charge traps reach thermal equilibrium and there is no further net emission of charges into the valence band. $A_{i}$ is the pre-exponential emission coefficient from an individual charge trap with energy $E_{T i}$ and decay time coefficient $\tau_{i}$. The use of a double-exponential term in Eq. (1) is necessary due to the two significantly different processes that occur during the relaxation step. Emission currents that originate from charges trapped at the metal-semiconductor interface are emitted back 

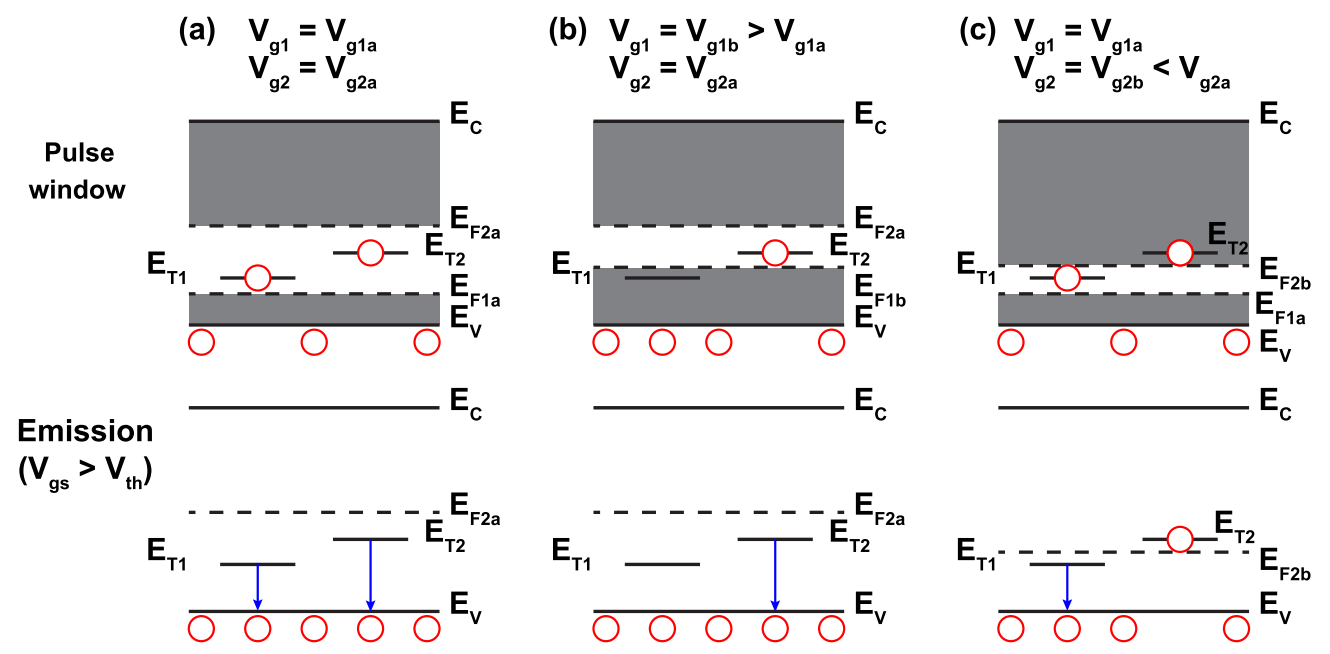

FIG. 2. Band diagrams showing the effects of changing $V_{g 1}$ and $V_{g 2}$ on the overall pulse window and the emission process when (a) $V_{g 1}=V_{g 1 a}$ and $V_{g 2}=V_{g 2 a}$, (b) $V_{g 1}$ is changed to $V_{g 1 b}$, and (c) $V_{g 2}$ is changed to $V_{g 2 b}$. In all the band diagrams, $E_{C}$ and $E_{V}$ correspond to the conduction and valence bands, respectively, $E_{T 1}$ and $E_{T 2}$ are the energy levels of two arbitrary trap states, and $E_{F 1 a(b)}$ and $E_{F 2 a(b)}$ are the Fermi levels at $V_{g 1 a(b)}$ and $V_{g 2 a(b)}$, respectively.

to the circuit on a relatively short timescale, while threshold voltage transient currents that are induced by charges trapped uniformly throughout the surface of the conductive channel need significantly longer times to recover. The fitted decay constants were found to be $\tau_{1} \sim 220 \mathrm{~s}$ and $\tau_{2} \sim 2200 \mathrm{~s}$ for the shallow and deep traps, respectively. These values are in good agreement with our earlier studies [1], giving a strong indication that the traps are inherent to this material/substrate system. The order-of-magnitude difference between the time constants means that the intermixing between the two exponential terms is negligible, and the two signals can be easily deconvoluted (see [27] for TVT experimental results).

\section{B. The energy dispersive spectroscopy technique}

The threshold voltage transient currents can provide a wealth of detail on the concentration of charge traps $N_{T}$ in the energy window defined by $V_{g 1}$ and $V_{g 2}$. The transient component of the current is given by

$$
\begin{aligned}
I_{d s, \text { trans }} & =A_{2} e^{-t / \tau_{2}} \\
& =\frac{q W \mu N_{T} V_{d s}}{L} e^{-t / \tau_{2}} .
\end{aligned}
$$

By assigning this term to the last term in Eq. (1), $N_{T}$ can be estimated using [28]

$$
N_{T}=\frac{L}{q W \mu} \frac{d A_{2}}{d V_{d s}} .
$$

It is important to clarify the physical meaning of $N_{T}$ in this equation, particularly in the context of the energetic and spatial boundaries of the traps included in this value. Contrary to the conventional method of estimating the trap concentration within depletion regions in bulk materials [29], two-dimensional materials are fully depleted in the vertical (van der Waals stack) direction [1]. Hence, considerations of the active volume defined by the transition length within the depletion region become irrelevant, as the entire volume is "active" in our analysis. On the other hand, the active-energy boundaries must be defined. To illustrate this, an arbitrary gate window is considered, defined by $V_{g 1 a}$ and $V_{g 2 a}$, as shown schematically in Fig. 2(a) with the nonshaded region of the pulse window highlighting the active trap energies. When the gate bias is set to $V_{g 1 a}$, the Fermi level $\left(E_{F}\right)$ shifts to a position close to the valence band edge and, in doing so, defines the shallowest gap state available for the occupation of holes. When the gate is pulsed back to $V_{g 2 a}, E_{F}$ is shifted to a position farther away from the valence band, defining the deepest gap state that can emit holes back to the valence band. Therefore, $N_{T}$ in this context is formally given by

$$
N_{T}=\int_{V_{g 2 a}}^{V_{g 1 a}} g_{T}\left(V_{g s}\right) d V_{g s}
$$

where $g_{T}\left(V_{g s}\right)$ is the density of trap states, per unit of $V_{g s}$. For a pulse window defined by $V_{g 1 a}=-35 \mathrm{~V}$ and $V_{g 2 a}=$ $-25 \mathrm{~V}$, the trap concentration was found from Eq. (3) to be $(6.7 \pm 3.2) \times 10^{10} \mathrm{~cm}^{-2}$, using the down-sweep mobility of $0.44 \mathrm{~cm}^{2} \mathrm{~V}^{-1} \mathrm{~s}^{-1}$.

By changing $V_{g 1}$, the concentration of charge traps changes in accordance with Eq. (4). First, when $V_{g 1}$ is increased to a less negative value $V_{g 1 b}$, corresponding to a less-shallow state, fewer traps are available for the occupation of holes, and thus fewer holes will be available for emission back to the valence band in the emission cycle. Indeed, when $V_{g 1 b}=-30 \mathrm{~V}$ and $V_{g 2}$ remains at $-25 \mathrm{~V}$, the trap concentration is found to be $(4.4 \pm 1.7) \times 10^{10} \mathrm{~cm}^{-2}$ and is, as expected, smaller than that of the original pulse window.

Following the same logic, if $V_{g 2}$ is now decreased to a more negative value $V_{g 2 b}$, corresponding to a shallower level, while $V_{g 1}$ reverts back to its original value $V_{g 1 a}$, fewer occupied traps will be able to emit captured holes back to the valence band, since the traps above $E_{F}$, as defined by $V_{g 2}$, will remain occupied, as schematically shown in Fig. 2(c). However, for $V_{g 2 b}=-30 \mathrm{~V}$ and $V_{g 1}=-35 \mathrm{~V}, N_{T}$ was found to be $(8.5 \pm 4.2) \times 10^{10} \mathrm{~cm}^{-2}$, an unexpected increase in $N_{T}$ when compared to the original pulse window.

To explain this increase in $N_{T}$, it is instructive to consider how it is calculated from the pre-exponential factors $d A_{2} / d V_{d s}$ 
(a)

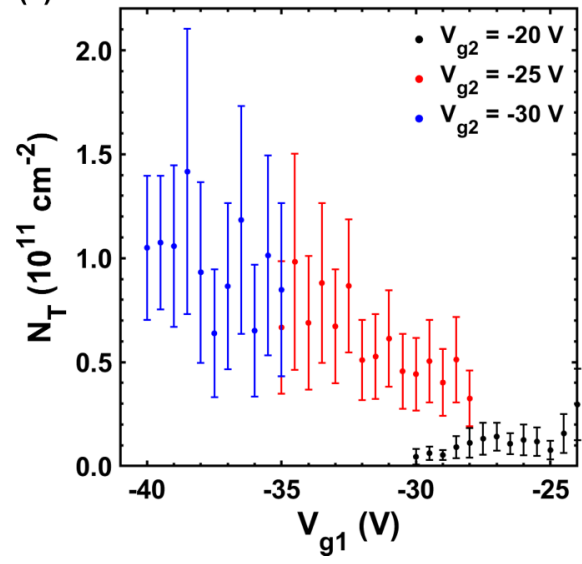

(b)

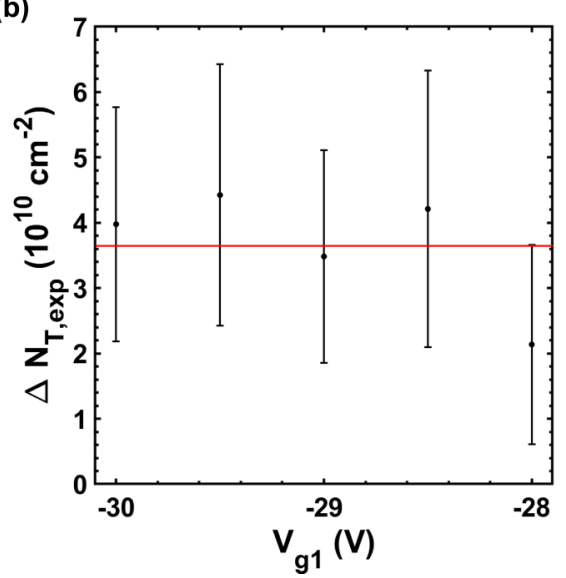

(c)

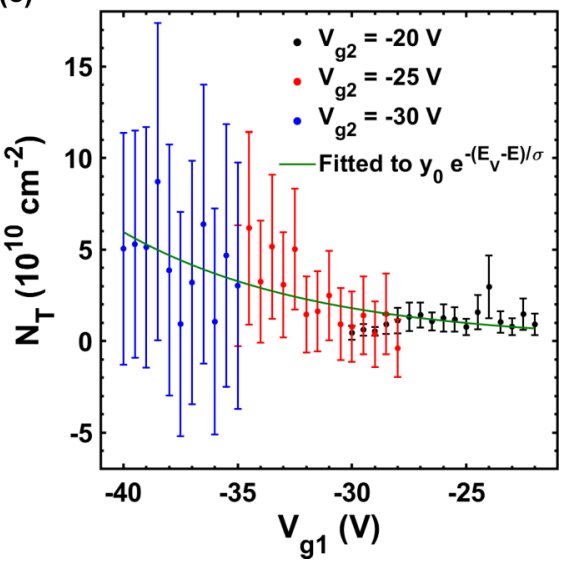

FIG. 3. (a) The concentration of charge traps as a function of $V_{g 1}$ with three different values of $V_{g 2}$. (b) The difference in trap concentration $\Delta N_{T, \exp }$ between $V_{g 2 a}$ and $V_{g 2 b}$. (c) The concentration of charge traps adjusted for the differences in $V_{g 2}$ using the average difference.

at a fixed $V_{g 1}$ for $V_{g 2 a}$ and $V_{g 2 b}$ :

$$
\begin{aligned}
& \frac{d A_{2 a}}{d V_{d s}}=\frac{q W \mu_{a}}{L} \int_{V_{g 2 a}}^{V_{g 1}} g_{T}\left(V_{g s}\right) d V_{g s}, \\
& \frac{d A_{2 b}}{d V_{d s}}=\frac{q W \mu_{b}}{L} \int_{V_{g 2 b}}^{V_{g 1}} g_{T}\left(V_{g s}\right) d V_{g s},
\end{aligned}
$$

where $\mu_{a(b)}$ is the effective mobility associated with $V_{g 2 a(b)}$. As can be seen, for the different values of $V_{g 2}, g_{T}\left(V_{g s}\right)$ is integrated over a different energy range, which will result in variations between $N_{T}$. Since the energy window defined by $V_{g 2 b}$ is smaller than the one defined by $V_{g 2 a}$, the integral in Eq. (5a) can be split into a sum of two integrals:

$$
\begin{aligned}
\int_{V_{g 2 a}}^{V_{g 1}} g_{T}\left(V_{g s}\right) d V_{g s}= & \int_{V_{g 2 a}}^{V_{g 2 b}} g_{T}\left(V_{g s}\right) d V_{g s} \\
& +\int_{V_{g 2 b}}^{V_{g 1}} g_{T}\left(V_{g s}\right) d V_{g s} \equiv \delta N_{T}+N_{T b},
\end{aligned}
$$

where $\delta N_{T}$ is the difference in trap concentration at $V_{g 1}$ when $V_{g 2}$ is varied between $V_{g 2 a}$ and $V_{g 2 b}$, and $N_{T b}$ is the trap concentration associated with $V_{g 2 b}$.

$A_{2}$ is also affected by the effective mobility of the charge carriers. As $V_{g 2}$ approaches the subthreshold region of the transfer curve, the current transport becomes limited by thermionic emission over the Schottky barrier [30], thus changing $A_{2}$. The changes in the device conductivity can be modeled, to a first approximation, as a linear perturbation in the mobility $\delta \mu$, so $\mu_{a}=\mu_{b}+\delta \mu$. It must be noted that it is not the mobility that changes with $V_{g s}$, but rather, that the injection of charges into the channel becomes limited by the formation of Schottky barriers at the contact. However, for simplicity of the model, these changes are incorporated as a small linear term in the effective mobility.

The difference in $d A_{2} / d V_{d s}$, so $\Delta\left(d A_{2} / d V_{d s}\right)=$ $d A_{2 a} / d V_{d s}-d A_{2 b} / d V_{d s}$, is then shown to be

$$
\Delta\left(d A_{2} / d V_{d s}\right)=\frac{q W}{L}\left(\delta \mu N_{T b}-\mu_{a} \delta N_{T}\right)
$$

(See [27] for a mathematical derivation of this model.)
Based on the above discussion, $\delta N_{T}<0$, so then if $\delta \mu \leqslant 0$, then the expected decrease in $\Delta A_{2}$ should be observed. However, when $\delta \mu>0$, the trend can be reversed provided $\delta \mu N_{T b}$ exceeds $\mu_{a} \delta N_{T}$, so that the change in $d A_{2} / d V_{d s}$ is shifted by a constant positive value $q W \delta \mu N_{T b} / L$.

The theoretical result is supported by comparing the measured difference in charge concentration $N_{T \text {,exp }}$ between $V_{g 2 a}$ and $V_{g 2 b}$. Figure 3(a) shows the data collected with $V_{g 2}$ values of -20 (black), -25 (red), and $-30 \mathrm{~V}$ (blue) without applying an offset. The series of differences between the red and black data where their $V_{g 1}$ values overlap, in Fig. 3(b), clearly shows a constant value within experimental error. This difference corresponds to $\delta N_{T}$ in Eq. (6), thus agreeing that the curves from different values of $V_{g 2}$ can be pieced together to yield the spectrum shown in Fig. 3(c).

To conclude this part, it should be noted that while the underlying physics of TVTS with different $V_{g 2}$ may be complex, the experimental act of piecing them together is a straightforward process. By way of demonstration, the trap density can be readily fitted by a decay exponential of the form $N_{T}=y_{0} e^{-\left(E_{V}-E\right) / \sigma}$, where $y_{0}$ is a proportionality factor, which is equal to the product of the DOS just at the edge of the valence band and $\sigma$ (the energy decay factor), in agreement with the expected tail states dispersion. From the fit, $y_{0}$ was found to be $\sim 5 \times 10^{8} \mathrm{~cm}^{-2}$ and $\sigma$ is $\sim 8.3 \mathrm{~V}$. While the fit to the exponential decaying profile serves only as a rough guide that demonstrates the validity of the proposed TVTS method, it can be seen in Fig. 3(c) that the trap concentration follows the proposed trend, within experimental error, providing further justification for the TVTS technique. However, the fit by itself serves no purpose in the analysis of the results.

\section{Extracting the density of trap states}

Finally, by combining the information on $N_{T}$ with details of the Fermi level $\left(E_{F}\right)$ obtained from KPFM measurements, shown in Fig. 4(a), the DOS within the band gap can be 
(a)

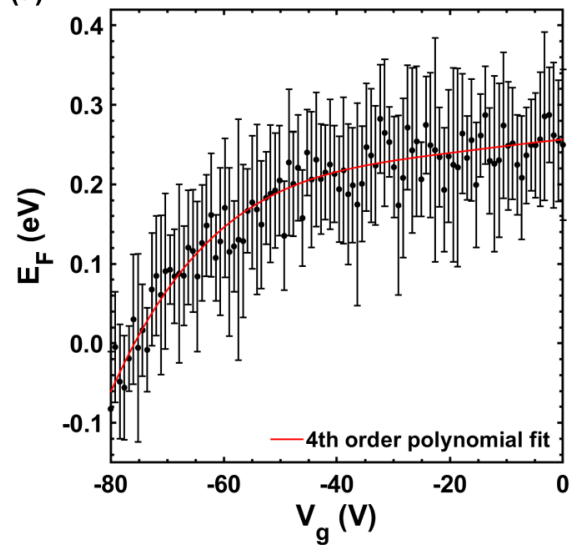

(b)

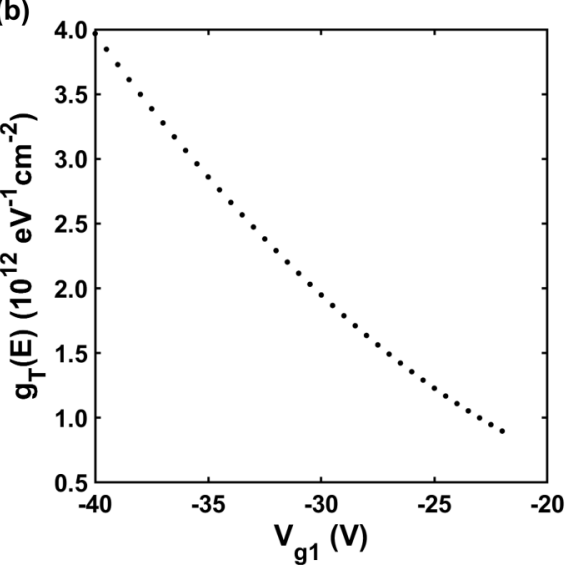

(c)

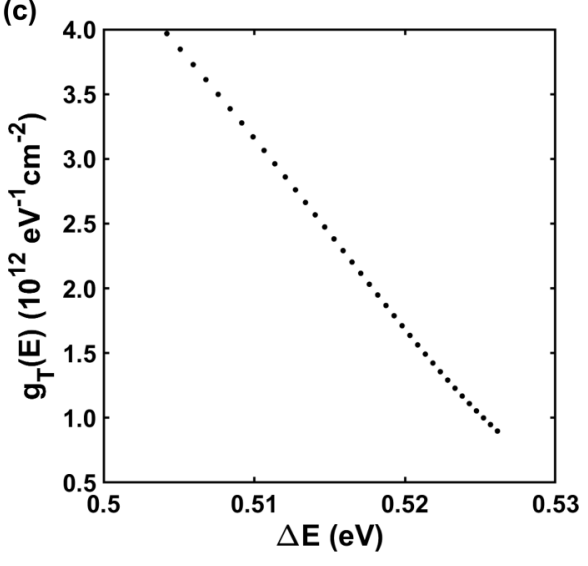

FIG. 4. (a) $E_{F}$ measured as a function of gate bias using KPFM and fitted with a fourth-order polynomial. (b) The density of trap states estimated from the fits of $N_{T}$ and $E_{F}$. (c) The density of trap states shown in (b) mapped to the energy location with respect to the top of the valance band.

estimated using

$$
g_{T}(E)=\frac{d N_{T}}{d V_{g 1}}\left(\frac{d E_{F}}{d V_{g 1}}\right)^{-1} .
$$

We note here that the value measured on the KPFM is the work function, which is defined as $\phi_{S}=\chi+\left(E_{C}-E_{F}\right)$, where $\chi$ is the electron affinity. Since $\chi$ and the conduction band minimum do not change with gate bias, to a first approximation, it is clear that

$$
-\frac{d \phi_{S}}{d V_{g 1}}=\frac{d E_{F}}{d V_{g 1}} .
$$

To allow for a continuous gradient of $E_{F}$ to be determined, the data were fitted with a fourth-order polynomial. The DOS was then found from the differentials of both fit curves, that for $N_{T}$ and the one for $E_{F}$, and is shown in Fig. 4(b). The charge neutrality point which manifests as the trough in current on the logarithm-scale trace in Fig. 1 is, to a good approximation, the location of the intrinsic Fermi level. Taking this value as a reference point, and the polynomial fit presented in Fig. 4(a) as a scaling function, a complete mapping of the gate bias to energetic location in the band gap is obtained. Using this mapping, the density of trap states has been evaluated with respect to the energetic depth of the trap, $\Delta E$, and is shown in Fig. 4(c).

The trend observed in the fitted data is in good agreement with theory, with the trap density of state showing what is most likely the onset of a midgap miniband as the Fermi level moves away from the valence band. However, this experiment has only probed a fraction of the band gap, so any defect states arising from specific contamination, such as water on the surface, which would show a more pronounced feature in the DOS [31], may be located outside of this range, as was discussed in light of the trap concentration.

\section{CONCLUSION}

In conclusion, the threshold voltage transient method has been extended into a fully spectroscopic technique allowing the concentration of trap states to be measured as a function of capture energy. This approach has been demonstrated using $\mathrm{MoTe}_{2}$ in ambient conditions, where it was found that small differences in mobility can increase the charge carrier concentration. Having merged together information gathered from sequential experiments done using different pulse windows, it is clear the trap concentration follows an expected decay trend as the energy shifts further from the band edge.

\section{ACKNOWLEDGMENTS}

N.J.T. and S.R. acknowledge DSTL grant scheme Sensing and Navigation Using Quantum 2.0 Technologies. I.A. acknowledges financial support from the European Commission Marie Curie Individual Fellowships (Grant No. 701704). S.R. and M.F.C. acknowledge financial support from EPSRC (Grants No. EP/J000396/1, No. EP/K017160/1, No. EP/K010050/1, No. EP/G036101/1, No. EP/M001024/1, and No. EP/M002438/1), from Royal Society International Exchanges Scheme 2016/R1, and from the Leverhulme Trust (grant title Quantum Drums and Room Temperature Quantum Electronics). O.K. and V.P. acknowledge funding from the European Union's Horizon 2020 research and innovation program under Grant Agreement GrapheneCore2 No. 785219 as well NMS funding within the 2DM X-theme project.
[1] I. Amit, T. J. Octon, N. J. Townsend, F. Reale, C. D. Wright, C. Mattevi, M. F. Craciun, and S. Russo, Adv. Mater. 29, 1605598 (2017).

[2] D. Kotekar-Patil, J. Deng, S. L. Wong, C. S. Lau, and K. E. J. Goh, Appl. Phys. Lett. 114, 013508 (2019).
[3] Q. H. Wang, K. Kalantar-Zadeh, A. Kis, J. N. Coleman, and M. S. Strano, Nat. Nanotechnol. 7, 699 (2012).

[4] H. S. Lee, S.-W. Min, M. K. Park, Y. T. Lee, P. J. Jeon, J. H. Kim, S. Ryu, and S. Im, Small 8, 3111 (2012). 
[5] N. Peimyoo, M. D. Barnes, J. D. Mehew, A. De Sanctis, I. Amit, J. Escolar, K. Anastasiou, A. P. Rooney, S. J. Haigh, S. Russo, M. F. Craciun, and F. Withers, Sci. Adv. 5, 8 (2019).

[6] M. M. Furchi, D. K. Polyushkin, A. Pospischil, and T. Mueller, Nano Lett. 14, 6165 (2014).

[7] J. D. Mehew, S. Unal, E. T. Alonso, G. F. Jones, S. F. Ramadhan, M. F. Craciun, and S. Russo, Adv. Mater. 29, 7 (2017).

[8] G. F. Jones, R. M. Pinto, A. De Sanctis, V. K. Nagareddy, C. D. Wright, H. Alves, M. F. Craciun, and S. Russo, Adv. Mater. 29, 7 (2017).

[9] Y. Y. Illarionov, G. Rzepa, M. Waltl, T. Knobloch, A. Grill, M. M. Furchi, T. Mueller, and T. Grasser, 2D Mater. 3, 035004 (2016).

[10] K. Lee, M. S. Oh, S. Mun, K. H. Lee, T. W. Ha, J. H. Kim, S.-H. K. Park, C.-S. Hwang, B. H. Lee, M. M. Sung, and S. Im, Adv. Mater. 22, 3260 (2010).

[11] J. Roh, J. H. Ryu, G. W. Baek, H. Jung, S. G. Seo, K. An, B. G. Jeong, D. C. Lee, B. H. Hong, W. K. Bae, J.-H. Lee, C. Lee, and S. H. Jin, Small 15, 1803852 (2019).

[12] M. J. Shearer, W. Li, J. G. Foster, M. J. Stolt, R. J. Hamers, and S. Jin, ACS Energy Lett. 4, 102 (2019).

[13] N. Liu, J. Baek, S. M. Kim, S. Hong, Y. K. Hong, Y. S. Kim, H.-S. Kim, S. Kim, and J. Park, ACS Appl. Mater. Interfaces 9, 42943 (2017)

[14] S. Tongay, J. Suh, C. Ataca, W. Fan, A. Luce, J. S. Kang, J. Liu, C. Ko, R. Raghunathanan, J. Zhou, F. Ogletree, J. Li, J. C. Grossman, and J. Wu, Sci. Rep. 3, 2657 (2013).

[15] D. V. Lang, J. Appl. Phys. 45, 3023 (1974).

[16] J. Bollmann and A. Venter, Physica B (Amsterdam) 535, 237 (2018).
[17] D. L. Losee, Appl. Phys. Lett. 21, 54 (1972).

[18] W. Shockley, Bell Syst. Tech. J. 28, 435 (1949).

[19] W. Shockley and W. T. Read, Phys. Rev. 87, 835 (1952).

[20] N. Al Saqri, J. F. Felix, M. Aziz, V. P. Kunets, D. Jameel, D. Taylor, M. Henini, M. S. Abd El-sadek, C. Furrow, M. E. Ware, M. Benamara, M. Mortazavi, and G. Salamo, Nanotechnology 28, 045707 (2017).

[21] M. Shafi, R. H. Mari, A. Khatab, M. Henini, A. Polimeni, M. Capizzi, and M. Hopkinson, J. Appl. Phys. 110, 124508 (2011).

[22] M. Shafi, R. H. Mari, A. Khatab, D. Taylor, and M. Henini, Nanoscale Res. Lett. 5, 1948 (2010).

[23] N. Sengouga, R. Boumaraf, R. H. Mari, A. Meftah, D. Jameel, N. Al Saqri, M. Azziz, D. Taylor, and M. Henini, Mater. Sci. Semicond. Process. 36, 156 (2015).

[24] T. P. Weiss, S. Nishiwaki, B. Bissig, S. Buecheler, and A. N. Tiwari, Phys. Chem. Chem. Phys. 19, 30410 (2017).

[25] J. A. Borsuk and R. M. Swanson, IEEE Trans. Electron Devices ED-27, 2217 (1980).

[26] Y. Nakano and T. Kachi, Appl. Phys. Lett. 79, 1631 (2001).

[27] See Supplemental Information at http://link.aps.org/ supplemental/10.1103/PhysRevB.100.165310 for $I_{d s}-V_{d s}$ response, charge trap characterization, and offsetting $N_{T}$ for changing $V_{g 2}$.

[28] S. M. Sze and K. K. Ng, Physics of Semiconductor Devices (John Wiley \& Sons, 2006).

[29] P. Blood and J. Orton, The Electrical Characterization of Semiconductors: Majority Carriers and Electron States (Academic Press, London, UK, 1992).

[30] N. J. Townsend, I. Amit, M. F. Craciun, and S. Russo, 2D Mater. 5, 025023 (2018).

[31] D. Oberhoff, K. P. Pernstich, D. J. Gundlach, and B. Batlogg, IEEE Trans. Electron Devices 54, 17 (2007). 